\title{
HURST PARAMETER ESTIMATION FOR EPILEPTIC SEIZURE DETECTION
}

\author{
IVAN OSORIO* AND MARK G. FREI ${ }^{\dagger}$
}

\begin{abstract}
Estimation of the Hurst parameter provides information about the memory range or correlations (long vs. short) of processes (time-series). A new application for the Hurst parameter, real-time event detection, is identified. Hurst estimates using rescaled range, dispersional and bridgedetrended scaled windowed variance analyses of seizure time-series recorded from human subjects reliably detect their onset, termination and intensity. Detection sensitivity is unaltered by signal decimation and window size increases. The high sensitivity to brain state changes, ability to operate in real time and small computational requirements make Hurst parameter estimation using any of these three methods well suited for implementation into miniature implantable devices for contingent delivery of anti-seizure therapies.
\end{abstract}

Key words: Hurst parameter, Epilepsy, Seizure Detection, Electrocorticogram, Implantable devices, Contingent therapy

1. Introduction. Epilepsy, a disabling disease, affects $1-2 \%$ of the American and industrialized world's population, and up to $10 \%$ of people in under-developed countries. As seizures are brief and relatively unpredictable, continuous EEG/ECoG monitoring is needed to implement new therapies, such as contingent electrical stimulation for seizure blockage, via implantable devices, in subjects with pharmaco-resistant epilepsies.

Hurst parameter [1] estimation has been applied to many natural (non-biological) [2] and also biological phenomena, such as neuron membrane channel kinetics [10], a fundamental functional operation of the brain. The behavior of membrane channels seems to exhibit long-term correlation ( $H>0.78$, implying "persistence") and the currents recorded through individual ion channels have self-similar properties, that is, they are fractals and may be best modeled using fractional Brownian motion. The fractal behavior may extend to the whole neuron as measured simultaneously across many channels. This raises the possibility that brain electrical processes may be fractal or self-similar, or that, at a minimum, useful information may be obtained from treating them as such in analyzing data or signals generated by these processes in the brain.

The Hurst parameter $(\mathrm{H})[1,2]$ may provide information about the behavior of continuous and discrete event time series and its estimation in the EEG/ECoG of

\footnotetext{
${ }^{*}$ Corresponding Author. Flint Hills Scientific, L. L. C., 5040 Bob Billings Pkwy, Suite A, Lawrence, KS 66049 USA and University of Kansas Medical Center, Kansas City, KS.

$\dagger$ †Flint Hills Scientific, L. L. C., 5040 Bob Billings Pkwy, Suite A, Lawrence, KS 66049 USA.
} 
humans with epilepsy may be useful for tracking seizures (Fig. 1) due to its relative simplicity. Of particular value for its implementation into miniature implantable or portable devices is the relative insensitivity of $\mathrm{H}$ estimates to signal decimation. If substantiated in applications to seizures, this characteristic would decrease the demands on the size and speed of digital signal processors allowing low power implementations. $\mathrm{H}$, also sometimes referred to as a rescaled range statistic, may be estimated using the relationship:

$$
\frac{R_{T}}{S_{T}} \sim T^{H} \text { as } T \rightarrow \infty
$$

where $\mathrm{R}$ is the range, $\mathrm{S}$ the standard deviation and $\mathrm{T}$, time. This leads to the Hurst parameter estimator:

$$
\hat{H} \sim \log \left(\frac{R_{T}}{S_{T}}\right) / \log (T) .
$$

2. Estimation of the Hurst parameter. While there are many available methods for the estimation of $\mathrm{H}[2,3,4,5,6,7,8,9,11]$, this paper will apply rescaled range $(\mathrm{R} / \mathrm{S})$ [2], dispersional analysis (DA) [5], and bridge-detrended scaled window variance (bdSWV) $[2,6]$, because they are well understood and yielded meaningful results in preliminary seizure data analyses.

The rescaled range statistic, used by Hurst in his study of rainfall along the Nile River, is defined according to the following equations:

$$
\begin{aligned}
& X^{*}(t)=\sum_{s=1}^{t} X(s), \\
& X^{2 *}(t)=\sum_{s=1}^{t} X^{2}(s), \\
& R(d)=\max _{0 \leq u \leq d}\left\{X^{*}(u)-(u / d) X^{*}(d)\right\}-\min _{0 \leq u \leq d}\left\{X^{*}(u)-(u / d) X^{*}(d)\right\}, \\
& S^{2}(d)=X^{2 *}(d) / d-\left(X^{*}(d) / d\right)^{2}, \\
& Z(d)=R(d) / S(d) .
\end{aligned}
$$

If there exists a real number $\mathrm{J}$, such that the limit as $\mathrm{d} \rightarrow \infty$ of $\mathrm{Z}(\mathrm{d}) / \mathrm{d}^{J}$ converges in distribution to a non-degenerate limit random variable, then the signal $X_{t}$ is said to have exponent $\mathrm{J}$ with constant $\mathrm{R} / \mathrm{S}$ pre-factor and the exponent $\mathrm{J}$ will be referred to as the Hurst parameter estimate for the signal $X_{t}$.

Dispersional Analysis (DA), a method introduced by Bassingthwaighte [5], is based on the variability of local averages of the signal over windows of length, $\tau$. It uses standard deviation, $\operatorname{SD}(\tau)$, of these local averages and repeats the calculation of $\mathrm{SD}(\tau)$ over many $\tau$ values. The estimate of $\mathrm{H}$ is then obtained by adding one to the slope of the regression of $\log \left[\operatorname{SD}(\tau) / \operatorname{SD}\left(\tau_{0}\right)\right]$ vs. $\log \left[\tau / \tau_{0}\right]$, where $\tau_{0}$ is a reference window size.

Bridge-detrended scaled windowed variance (bdSWV), [2,6], divides a signal into windows of size $\tau$ and bridge-detrends it (i.e., subtracts the line connecting the first 
and last points in the window, then multiplies by a parabolic windowing function) and computes the standard deviation in each of the windows. The average of the standard deviations is then computed and used to estimate $\mathrm{H}$ as the slope of the regression of $\log \left[\mathrm{SD}(\tau) / \mathrm{SD}\left(\tau_{0}\right)\right]$ vs. $\log \left[\tau / \tau_{0}\right]$, where $\tau_{0}$ is a reference window size.

3. Methods and Materials. R/S, DA and bdSWV were applied to twenty 10minute segments of ECoG data from 10 subjects ( 2 per subject), with each segment containing a seizure. The data were collected from patients with pharmaco-resistant mesial temporal lobe seizures who underwent evaluation for epilepsy surgery at the University of Kansas Comprehensive Epilepsy Center. It was recorded using multiple contact depth electrodes (Ad-Tech, Racine, WI) placed in the amygdala-hypocampal regions and correctness of electrode placement was assessed with MRI. The signal was sampled at a rate of $240 \mathrm{~Hz}$, amplified to a dynamic range of $\pm 300 \mu \mathrm{V}$, and digitized with 10 bits of precision with $0.59 \mu \mathrm{V} /$ bit using commercially available devices (Nicolet, Madison, WI). The recordings were deemed of good technical quality and suitable for analysis. Additionally, analysis of a continuous recording (85 hours containing 62 seizures) from one subject was performed.

4. Results. Estimation of the Hurst parameter using R/S, DA and bdSWV in a moving window of data enables detection of certain changes in brain state, such as those associated with seizures. Specifically, H accurately detected (as compared to expert visual analysis, the "gold standard") onset and termination (duration) of all twenty seizures from 10 subjects, and provided an indirect estimate of intensity. Moreover, the Hurst parameter estimates appear to be sensitive to certain signal changes (which we refer to as "precursors") indicative of probable/impending seizure onset (Fig 2).

Estimation of $\mathrm{H}$ using R/S, DA and bdSWV showed comparable sensitivity and specificity for seizure detection. Regardless of the pre-seizure $H$ values (which are different for each method), seizure onset is characterized in all of them by the sudden, simultaneous and marked drop in value, which remains low for the duration of the seizure (FIG. 3). H increases rapidly at the end of the seizure, remaining above preseizure values for about $40 \mathrm{~s}$, , a period corresponding to the immediate post-ictal state; the return of $\mathrm{H}$ to pre-seizure values marks the end of this state and the beginning of the interictal period. The bdSWV characterized seizures as a transient increase in "antipersistence," whereas the others typically showed a decrease in "persistence" (Fig. 3).

Using the R/S method, $\mathrm{H}$ was estimated in moving $2 \mathrm{~s}$, non-overlapping windows, from 62 segments of data each containing one seizure (Fig 4A) and from 61 nonseizure segments of equal duration recorded midway between the seizures (Fig 4B). 
The temporal evolution of the distribution (illustrated using its decile values) of $\mathrm{H}$ shows an appreciable increase approximately $15 \mathrm{~s}$ before the electrographic seizure onset (at time 0; Fig 4A), remaining unchanged on segments without seizures (Fig $4 \mathrm{~B})$. That these changes in $\mathrm{H}$ estimates are seen in all deciles, indicates the sensitivity of the entire distribution of this parameter to relevant signal changes.

To test the robustness of $\mathrm{H}$ to decimation, ECoG was increasingly down-sampled from an original rate of $240 \mathrm{~Hz}$ to $1 \mathrm{~Hz}$. Using R/S, H estimates were obtained from moving $10 \mathrm{~s}$ windows (sliding $1 \mathrm{~s}$ at a time) of ECoG containing a seizure (onset: $\mathrm{t}=300 \mathrm{~s}$.; end: $\mathrm{t}=400 \mathrm{~s}$; postictal period until $\mathrm{t}=435 \mathrm{~s}$.). Each trace (Fig. 5 ) is the output of the signal decimated to the sampling rate annotated on the y-axis. The ability of $\mathrm{H}$ estimates to detect the beginning of the seizure remains essentially unaffected by decimation all the way down to the $15 \mathrm{~Hz}$ sampling rate. Moreover, the ability to detect seizure onset is preserved even with sampling rates as low as $1 \mathrm{~Hz}$., but for sampling rates below $15 \mathrm{~Hz}$., the direction of $\mathrm{H}$ change reverses (increases) at onset. Moreover, increases in window size from 1s/window to 10s/window (Fig. 6) do not impair detection of onset and termination using $\mathrm{H}$ estimates.

The ability of $\mathrm{H}$ to detect brain state changes is further demonstrated with the following experimental paradigm: Administration of 3 mercapto-propionic acid (3MPA) to rats causes seizures after a relatively constant latency. FIG. 7 shows the increase in $\mathrm{H}$ estimate, obtained from the ECoG of an animal injected with 3-MPA at time 0 (x-axis). The state change (from non-seizure to seizure) manifests with an increase in $\mathrm{H}$ values, which precedes the onset of seizures (annotated by a vertical line) by several minutes, thus predicting the state change.

5. Discussion. Estimation of the Hurst parameter on ECoG recorded from epileptic brains and the results obtained in this investigation, do not imply or infer a priori knowledge about the presence or absence of long-range dependencies in this signal. Furthermore these results do not provide insight into the dynamics of seizures or lead to their classification as fractional Gaussian noise, fractional Brownian motion or as unclassifiable, since the required steps [7] to accomplish this were not performed. Nonetheless, these results demonstrate that estimation of the Hurst parameter using $\mathrm{R} / \mathrm{S}$, DA or bdSWV, reliably detect the onset and termination (duration) and intensity of certain brain state changes, in this case seizures. Its robustness to decimation is a valuable feature for implementation into miniaturized, low-power implantable devices. The decimation in the example provided here would correspond to a factor of 16 improvement in number of required operations and amount of system memory required to detect the seizure, as compared to the original $240 \mathrm{~Hz}$ sampling rate.

Certain findings of this research raise questions about the signal's features to 
which R/S, DA and bdSWV are sensitive. Despite their mathematical similarity, the estimates they yield when applied to the same signal, are quantitatively and qualitatively different (Fig 3). H estimates of the interictal state based on R/S and DA, indicate "persistence", the estimates using bdSWV point to "antipersistence", while all three show a nearly simultaneous decrease in $\mathrm{H}$ (in reference to interictal) throughout the seizure. Of interest is also the reversal in the direction of change in $\mathrm{H}$ values, as a function of sampling rate (Fig. 5), without affecting its ability to detect state changes. This suggests that, at least when applied to short segments and short windows, it may provide confounding dynamical information about the signal in question. A systematic investigation of the effects of sampling rate and window size may provide valuable knowledge about the behavior of these and other Hurst estimators when applied to time series several orders of magnitude shorter than those to which they have traditionally been applied. This is particularly relevant for biological time series such as EKG and EEG to which Hurst estimators are applied to probe into their dynamics, without sound basis.

To summarize, the Hurst parameter detects in real time certain brain state changes, such as epileptic seizures, and is well suited for implementation into lowpower implantable devices for contingent delivery of anti-seizure therapies. The validity of applying this method to biological time series for purposes other than event detection deserves investigation.

6. Figure legends. FIG. 1 shows an electrocorticogram (ECoG) signal containing a seizure with electrographic and clinical seizure onsets (EO and CO, respectively) and subject activation of an event button (EB) annotated.

FIG. 2 displays 62 ECoG segments each containing seizure precursors, with several of these further evolving to higher frequency electrographic seizures. This illustrates the utility of Hurst parameter estimation for detection, quantification, and classification of different state changes. $\mathrm{H}$ estimates are represented by color changes in the traces, with numerical values indicated in the legend to the right.

FIG. 3 shows the changes in $\mathrm{H}$ parameter estimates (y-axis) obtained using three different methods (R/S, DA. bdSWV) applied to a moving window of human ECoG containing a seizure and postictal period (annotated by vertical bars).

FIG. 4A shows the evolution of the distribution of $\mathrm{H}$ parameter estimates (illustrated using 10 percentile divisions) with an increase approximately $15 \mathrm{~s}$ before electrographic seizure onset (time 0 in $\mathrm{x}$-axis) as marked using expert visual analysis. Fig. 4B shows the evolution of the distribution of $\mathrm{H}$ parameter estimates obtained from interictal segments of the same length recorded midway between the seizures.

FIG. 5 illustrates the robustness of Hurst parameter estimates as the ECoG signal from which they are obtained is markedly decimated from an original sampling rate 
of $240 \mathrm{~Hz}$ to various lower sampling rates ranging from $120 \mathrm{~Hz}$ down to $1 \mathrm{~Hz}$.

FIG. 6 illustrates the robustness of Hurst parameter estimates for seizure detection as the window size used for computing the estimates is varied.

FIG. 7 shows the increase in $\mathrm{H}$ estimate obtained from the ECoG of an animal to whom a convulsant substance (3-mercaptopropionic acid) was injected at time 0 . The state change (from non-seizure to seizure) manifests as an increase in $\mathrm{H}$ values that precedes the onset of seizures (annotated by a vertical line) by several minutes, thus predicting the state change.

Fig. 1
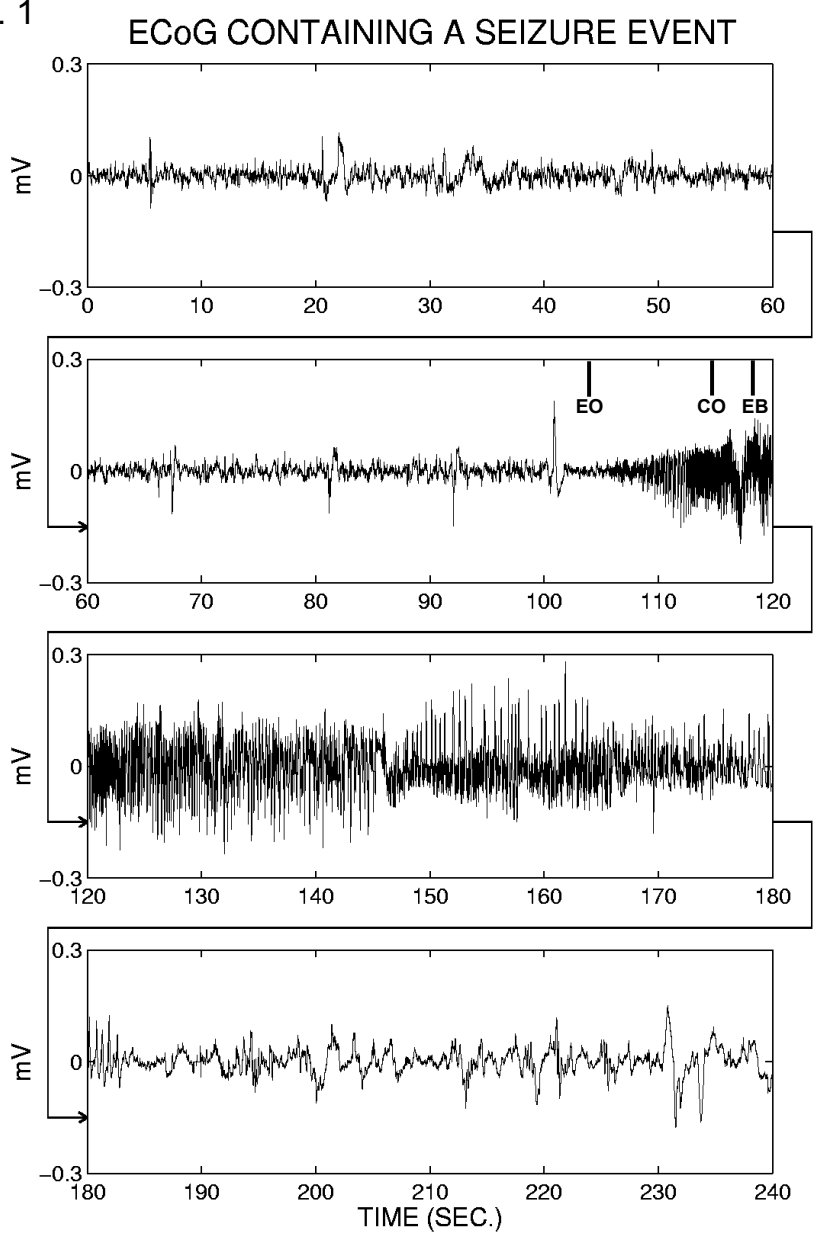
Fig. 2
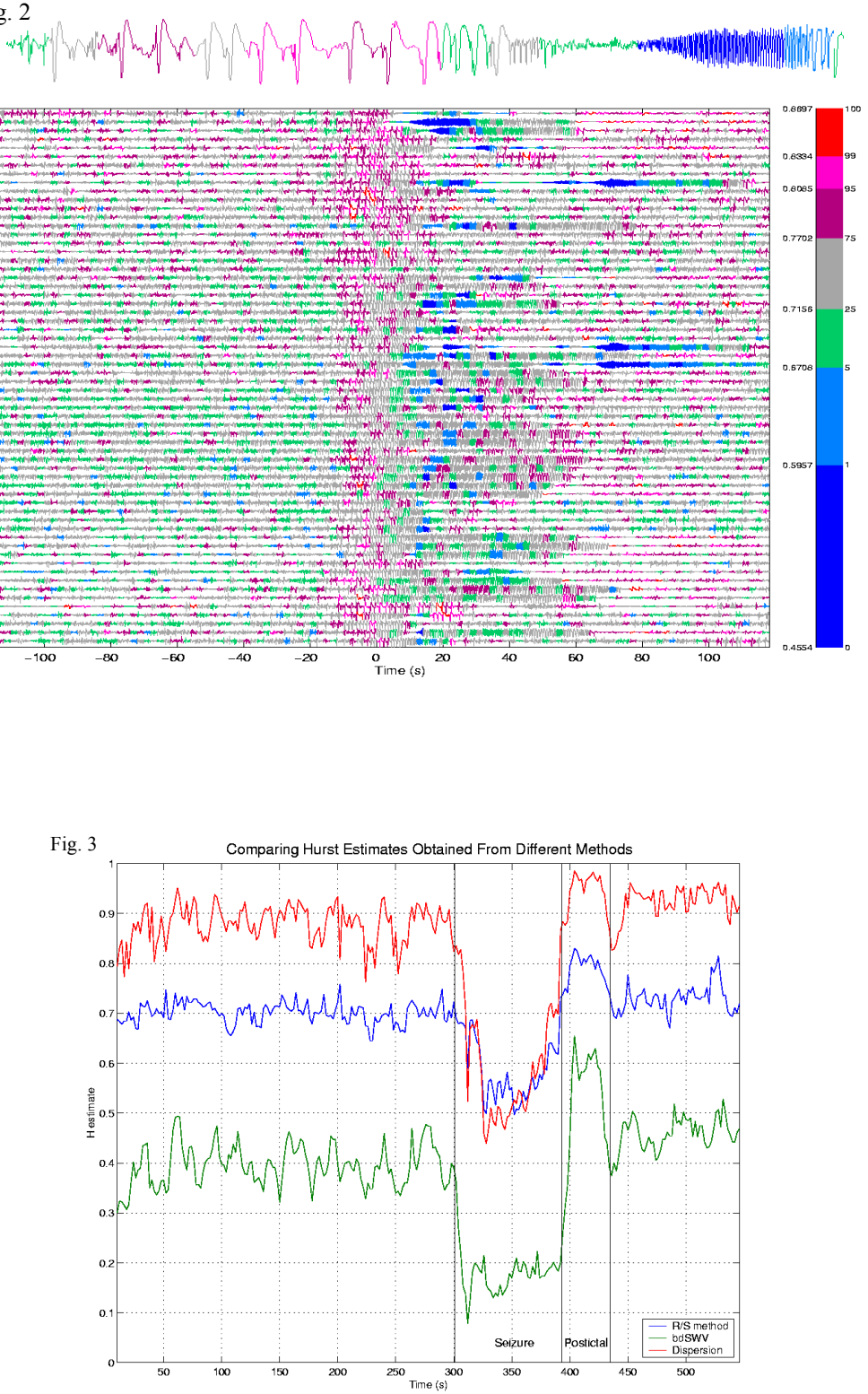

Acknowledgement. The authors would like to thank Professor Tyrone Duncan for many stimulating discussions about the Hurst parameter. 
Fig. 4
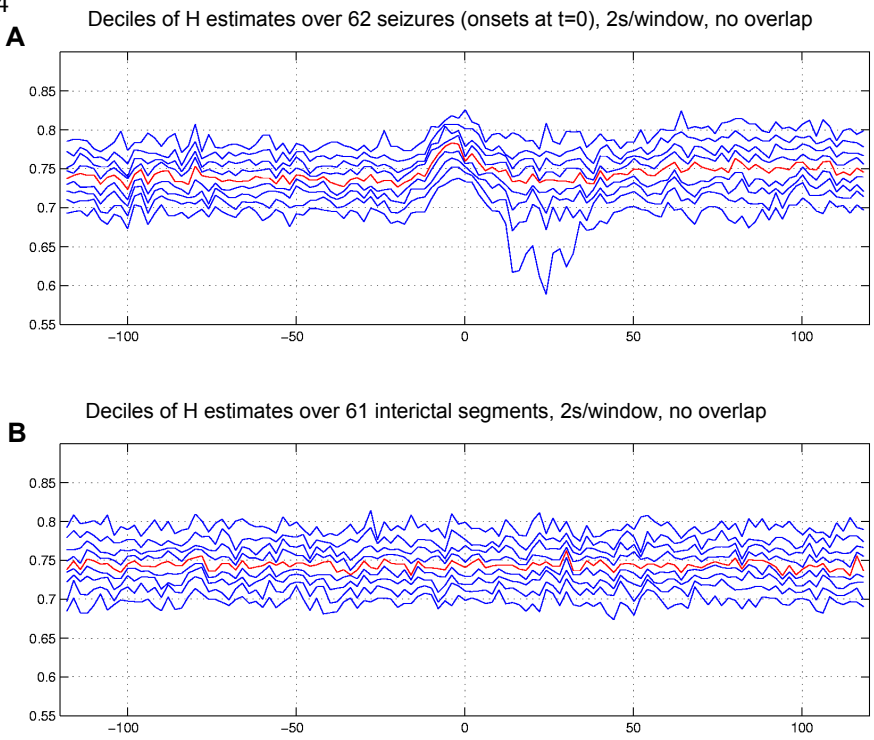

REFERENCES

[1] H. E. Hurst Long-term capacity storage of reservoirs. Trans Amer Soc Civil Engineers. 1951;116:770-99.

[2] B. B. Mandelbrot, The fractal Geometry of Nature, 1983, Freeman, San Francisco.

[3] B. B. Mandelbrot and J. R. Wallis, Robustness of the rescaled range $(R / S)$ in the measurement of noncycling long run statistical dependence, Water Resources Research, 5(1969), pp. 967-988.

[4] J. Beran, Statistics for Long-Memory Processes, Chapman \& Hall/CRC 1994 Boca Raton, London, New York, Washington, DC.

[5] J. E. Bassignthwaighte And G. M. RAYmond, Evaluation of the dispersional analysis method for fractal time series, Ann Biomed Eng, 23(1995), pp. 491-505.

[6] M. J. Cannon, D. B. Percival, D. C. Caccia, and M. J. Raymond, Bassingthwaigthe Je. Evaluating scaled window variance methods for estimating the Hurst coefficient of time series, Physica A, 241(1997), pp. 606-626.

[7] A. Eke, P. Herman, J. E. Bassingthwaigthe, and G. M. Raymond, D. B. Percival, M. Cannon, I. Balla, and C. Ikrenyi, Physiological time series: Distinguishing fractal noises from motions, Eur J Physiol, 439(2000), pp. 403-415.

[8] H. Kettani, A novel approach to the estimation of the long range dependence parameter, Ph.D. thesis 2002 University of Wisconsin, Madison.

[9] H. Kettani and J. A. Gubner, A novel approach to the estimation of the Hurst parameter in self-similar traffic, Proceeding of the Conference on local computer networks Tampa, FL, November 2002.

[10] S. B. Lowen, L. S. Liebovitch, And J. A. White, Fractal ion-channel behavior generates fractal firing patterns in neuronal models, Phys Rev E Stat Phys Plasmas Fluids Relat 
Fig. 5

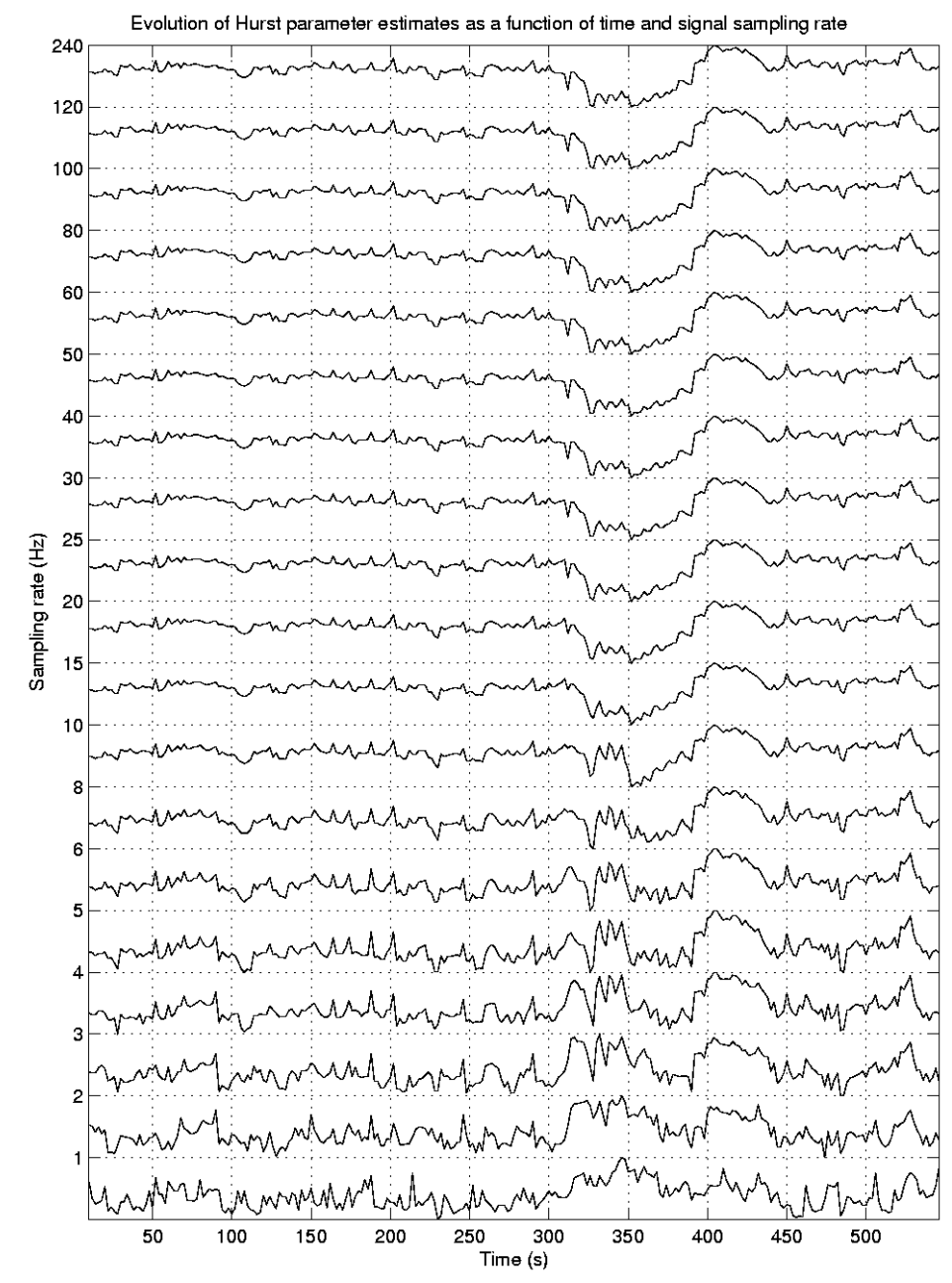

Interdiscip Topics, 59(5 Pt B), 1999, pp. 5970-80.

[11] P. Abry AND D. Veitch, Wavelet analysis of long-range dependent traffic, IEEE Trans Information Theory, 44(1998), pp. 2-15. 


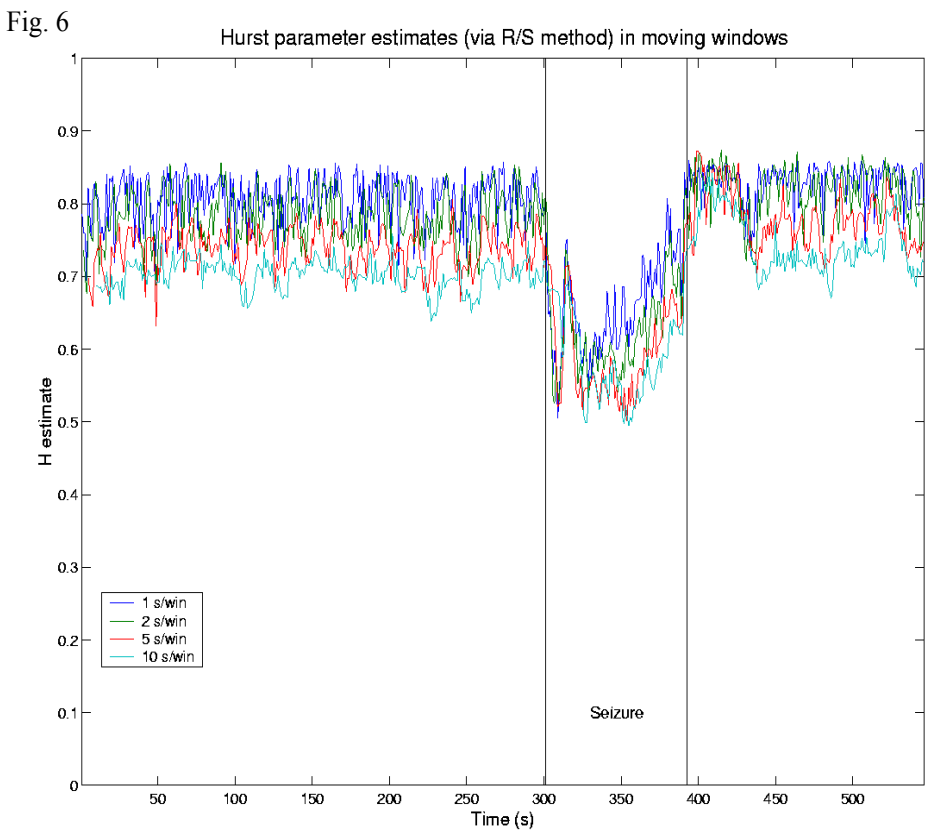

Fig. 7
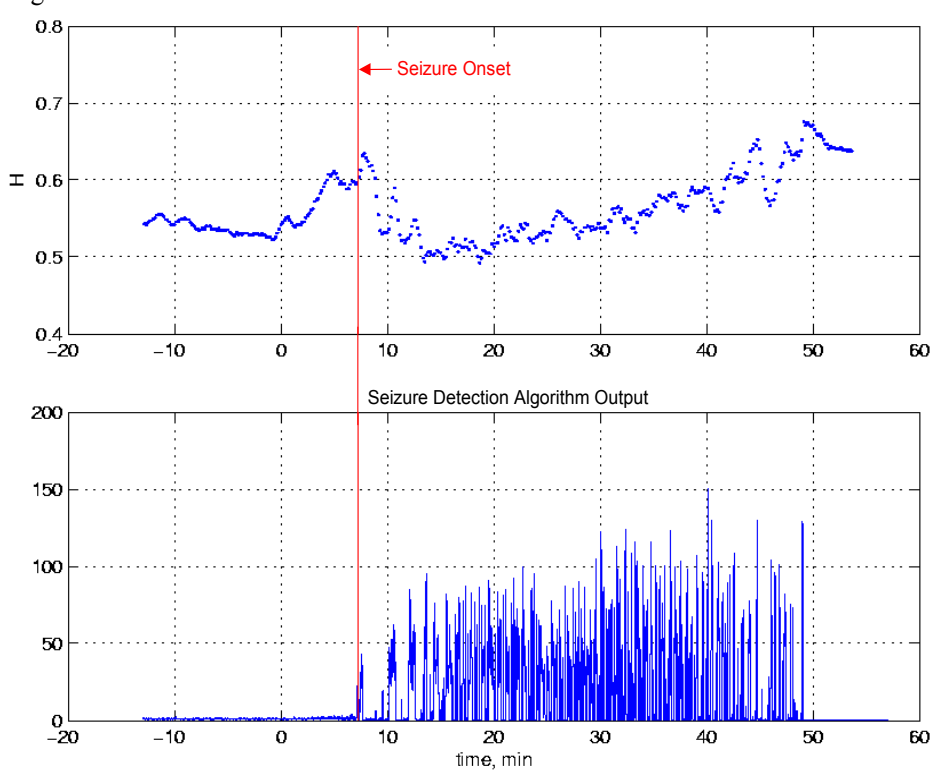